PROCEEDINGS OF THE

AMERICAN MATHEMATICAL SOCIETY

Volume 127, Number 6, Pages 1683-1692

S 0002-9939(99)04705-X

Article electronically published on February 11, 1999

\title{
THE FURUTA INEQUALITY WITH NEGATIVE POWERS
}

\author{
KÔTARÔ TANAHASHI
}

(Communicated by Palle E. T. Jorgensen)

\begin{abstract}
Let $A, B \in B(H)$ be bounded linear operators on a Hilbert space $H$ satisfying $O \leq B \leq A$. Furuta showed the operator inequality $\left(A^{r} B^{p} A^{r}\right)^{\frac{1}{q}} \leq$ $A^{\frac{p+2 r}{q}}$ as long as positive real numbers $p, q, r$ satisfy $p+2 r \leq(1+2 r) q$ and $1 \leq q$. In this paper, we show this inequality is valid if negative real numbers $p, q, r$ satisfy a certain condition. Also, we investigate the optimality of that condition.
\end{abstract}

\section{INTRODUCTION}

Let $A, B$ be bounded linear operators on a Hilbert space $H$. The following operator inequality is well-known as the Heinz inequality. (In the case where $p=\frac{1}{2}$, the inequality is called the Löwner inequality, and it is known that there are counterexamples in the case where $p>1$. See [1] for $p=2$ and [12, p. 465] for $p>1$.)

Proposition 1 ([10],[11]). $O \leq B \leq A$ implies $B^{p} \leq A^{p}$ for all $p \in[0,1]$.

Concerning the Heinz inequality, Chan and Kwong [1] conjectured that $O \leq B \leq$ $A$ will imply $B^{2} \leq\left(B A^{2} B\right)^{\frac{1}{2}}$ and Furuta gave an affirmative answer as follows.

Proposition 2 ([4]). $O \leq B \leq A$ implies

$$
\left(A^{r} B^{p} A^{r}\right)^{\frac{1}{q}} \leq A^{\frac{p+2 r}{q}}
$$

and

$$
B^{\frac{p+2 r}{q}} \leq\left(B^{r} A^{p} B^{r}\right)^{\frac{1}{q}}
$$

as long as positive real numbers $p, q, r$ satisfy

$$
p+2 r \leq(1+2 r) q \text { and } 1 \leq q .
$$

The range of $p, q, r$ which satisfy the condition (3) is as in Figure 1 and, in [13], the author proved that the condition (3) is optimal for the validity of the Furuta inequality by using $2 \times 2$ matrices.

The Furuta inequality has many applications. In the operator mean theory, the Furuta inequality was generalized to the grand Furuta inequality [8] and it extends the Ando-Hiai log-majorization theory. Also, the Furuta inequality was used in the $p$-hyponormal operator theory ([2], [9]) and the relative entropy theory ([6], [7]).

Recently, it is known that the Furuta inequality is valid for some negative real numbers $p, q, r$ by Yoshino [14] and Fujii, Furuta, Kamei [3]. And, in this paper,

Received by the editors September 29, 1995 and, in revised form, June 12, 1996, August 6, 1996, October 23, 1996, April 3, 1997, and September 4, 1997.

1991 Mathematics Subject Classification. Primary 47B15.

Key words and phrases. Löwner-Heinz inequality, the Furuta inequality, positive operator. 


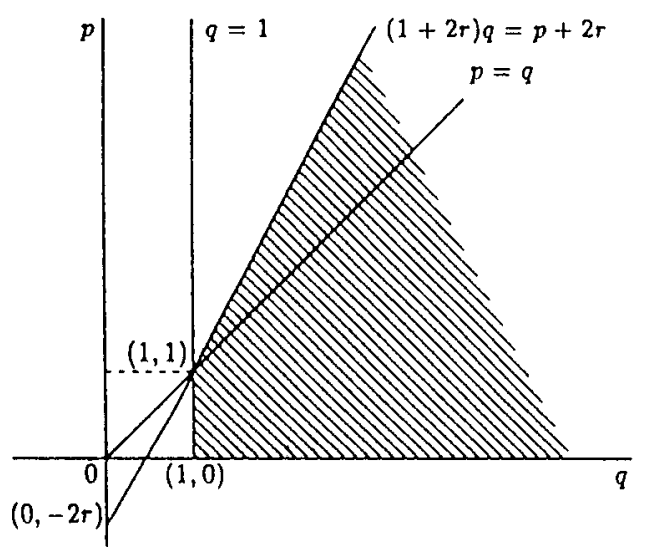

Figure 1

we shall consider the range of $p, q, r$ for which the Furuta inequality is valid and investigate its optimality by using the same technique as in [13].

\section{Results}

Let $A, B \in B(H)$ be invertible operators on $H$ satisfying $O \leq B \leq A$. In the Furuta inequality, if $p=0$, then $A^{0}=B^{0}=I$ and the inequalities (1) and (2) become the equalities. Also, if $r=0$, then the inequalities (1) and (2) reduce to the Löwner-Heinz inequality. Hence we may assume $p \neq 0$ and $r \neq 0$ in this paper. Since $O \leq B \leq A$ implies $O \leq A^{-1} \leq B^{-1}$, the validity of (1) is equivalent to the validity of (2). By a simple observation, the problem to find all real numbers $p, q, r$ guaranteeing the validity of the Furuta inequality is reduced to the case where $0<p, 0<q$ and $r<0$.

Theorem 3. Let $A, B \in B(H)$ be invertible operators satisfying $O \leq B \leq A$. Let $0<p \leq 1,0<q \leq 1$ and $-1 \leq 2 r<0$. Then

$$
\left(A^{r} B^{p} A^{r}\right)^{\frac{1}{q}} \leq A^{\frac{p+2 r}{q}}
$$

as long as real numbers $p, q, r$ satisfy

$$
-2 r(1-q) \leq p \leq q-2 r(1-q)
$$

and

$$
\frac{-2 r(1-q)-q}{1-2 q} \leq p \leq \frac{-2 r(1-q)}{1-2 q} .
$$

We need the following lemma to show Theorem 3 .

Lemma 4 ([5]). Let $A, B \in B(H)$ be positive operators and let $\lambda$ be a real number. Then

$$
(A B A)^{\lambda}=A B^{\frac{1}{2}}\left(B^{\frac{1}{2}} A^{2} B^{\frac{1}{2}}\right)^{\lambda-1} B^{\frac{1}{2}} A .
$$

Proof of Theorem 3. Let $0<p \leq 1,0<q \leq 1$ and $-1 \leq 2 r<0$. 
First, assume $\frac{1}{2} \leq q \leq 1$ and $p, q, r$ satisfy (4). (We remark that $p, q, r$ satisfy (5) automatically in this case.) Then $O \leq A^{2 r} \leq B^{2 r}$ by the Heinz inequality. Since

$$
0 \leq \frac{1}{q}-1 \leq 1
$$

and

$$
0 \leq \frac{p+2 r}{q}-2 r \leq 1
$$

we have

$$
\begin{aligned}
\left(A^{r} B^{p} A^{r}\right)^{\frac{1}{q}} & =A^{r} B^{\frac{p}{2}}\left(B^{\frac{p}{2}} A^{2 r} B^{\frac{p}{2}}\right)^{\frac{1}{q}-1} B^{\frac{p}{2}} A^{r} \\
& \leq A^{r} B^{\frac{p}{2}}\left(B^{\frac{p}{2}} B^{2 r} B^{\frac{p}{2}}\right)^{\frac{1}{q}-1} B^{\frac{p}{2}} A^{r}=A^{r} B^{\frac{p+2 r}{q}-2 r} A^{r} \\
& \leq A^{r} A^{\frac{p+2 r}{q}-2 r} A^{r}=A^{\frac{p+2 r}{q}}
\end{aligned}
$$

by Lemma 4 and the Heinz inequality.

Second, assume $\frac{1}{3} \leq q<\frac{1}{2}$ and $p, q, r$ satisfy (4) and (5). Let

$$
p^{\prime}=2 r, q^{\prime}=\frac{q}{1-q}, r^{\prime}=\frac{p}{2}
$$

and let

$$
p^{\prime \prime}=-p^{\prime}, q^{\prime \prime}=q^{\prime}, r^{\prime \prime}=-r^{\prime}
$$

Then

$$
0<p^{\prime \prime} \leq 1, \frac{1}{2} \leq q^{\prime \prime}<1,-\frac{1}{2} \leq r^{\prime \prime}<0
$$

and $p^{\prime \prime}, q^{\prime \prime}, r^{\prime \prime}$ satisfy

$$
-2 r^{\prime \prime}\left(1+q^{\prime \prime}\right)-q^{\prime \prime} \leq p^{\prime \prime} \leq-2 r^{\prime \prime}\left(1+q^{\prime \prime}\right)
$$

and

$$
-2 r^{\prime \prime}\left(1-q^{\prime \prime}\right) \leq p^{\prime \prime} \leq q^{\prime \prime}-2 r^{\prime \prime}\left(1-q^{\prime \prime}\right) .
$$

Hence, by the same arguments as in the case where $\frac{1}{2} \leq q \leq 1$, we have

$$
\left(A^{r^{\prime \prime}} B^{p^{\prime \prime}} A^{r^{\prime \prime}}\right)^{\frac{1}{q^{\prime \prime}}} \leq A^{\frac{p^{\prime \prime}+2 r^{\prime \prime}}{q^{\prime \prime}}},
$$

and

$$
A^{\frac{p^{\prime}+2 r^{\prime}}{q^{\prime}}} \leq\left(A^{r^{\prime}} B^{p^{\prime}} A^{r^{\prime}}\right)^{\frac{1}{q^{\prime}}}
$$

It is known that (6) implies

$$
\left(B^{r^{\prime}} A^{p^{\prime}} B^{r^{\prime}}\right)^{\frac{1}{q^{\prime}}} \leq B^{\frac{p^{\prime}+2 r^{\prime}}{q^{\prime}}}
$$

and hence

$$
\left(B^{\frac{p}{2}} A^{2 r} B^{\frac{p}{2}}\right)^{\frac{1}{q}-1} \leq\left(B^{\frac{p}{2}} B^{2 r} B^{\frac{p}{2}}\right)^{\frac{1}{q}-1} .
$$

Since $0 \leq \frac{p+2 r}{q}-2 r \leq 1$, we have

$$
\left(A^{r} B^{p} A^{r}\right)^{\frac{1}{q}} \leq A^{\frac{p+2 r}{q}}
$$

by the same arguments as in the case where $\frac{1}{2} \leq q \leq 1$. 
Similarly we can show the inequality (1) in the case where $\frac{1}{n+1} \leq q<\frac{1}{n}$ ( $n=$ $3,4,5, \cdots)$ and $p, q, r$ satisfy (4) and (5) because

$$
\frac{-2 r(1-q)-q}{1-2 q} \leq-2 r(1+q)-q<-2 r(1+q) \leq \frac{-2 r(1-q)}{1-2 q}
$$

if $0<2 q \leq 1$ and $-1 \leq 2 r<0$. Thus the proof is complete.

Theorem 3 shows the validity of the Furuta inequality as long as $p, q, r$ satisfy any one of the following conditions (I), (II), (III), (IV).

Condition (I). $-\frac{1}{4} \leq r<0$ and

$$
\left\{\begin{array}{lll}
q \geq \frac{p+2 r}{2 r}, & \text { if } & 0<p<-2 r \\
q>0, & \text { if } & p=-2 r \\
q \geq \frac{p+2 r}{2 p+2 r}, & \text { if } & -2 r<p \leq \frac{1}{2} \\
q \geq \frac{p+2 r}{1+2 r}, & \text { if } & \frac{1}{2}<p \leq 1 .
\end{array}\right.
$$

Condition (II). $-\frac{1}{2}<r<-\frac{1}{4}$ and

$$
\left\{\begin{array}{lll}
q \geq \frac{p+2 r}{2 r}, & \text { if } & 0<p<\frac{1}{2}, \\
q \geq \frac{p+2 r}{2 p+2 r-1}, & \text { if } & \frac{1}{2} \leq p<-2 r, \\
q>0, & \text { if } & p=-2 r, \\
q \geq \frac{p+2 r}{1+2 r}, & \text { if } & -2 r<p \leq 1 .
\end{array}\right.
$$

Condition (III). $r=-\frac{1}{2}$ and

$$
\left\{\begin{array}{lll}
q \geq \frac{p-1}{-1}, & \text { if } & 0<p<\frac{1}{2}, \\
q \geq \frac{1}{2}, & \text { if } & \frac{1}{2} \leq p<1 \\
q>0, & \text { if } & p=1 .
\end{array}\right.
$$

Condition (IV). $r<-\frac{1}{2}$ and

$$
q \geq 1, \quad \text { if } \quad 0<p \leq 1 .
$$

Remark. Yoshino [14] showed the validity of the inequality (1) as long as $p, q, r$ satisfy $-1<2 r<0,1 \leq 2 q,(1+2 r) q \leq p+2 r, 0<p \leq 1$ or the condition (IV). Moreover, Fujii, Furuta, Kamei [3] showed the validity of the inequality (1) as long as $p, q, r$ satisfy $-1<2 r<0,1 \leq 2 p \leq 2,0 \leq p+2 r,(1+2 r) q \leq p+2 r$.

In the sequel, we shall show that the condition (I) with $0<p \leq-2 r, \frac{1}{2} \leq p \leq 1$ and the conditions (II), (III), (IV) are optimal. Unfortunately, we remark that this is a partial answer for our problem and it is still an open question whether the Furuta inequality is valid as long as $p, q, r$ satisfy

$$
-\frac{1}{4}<r<0, \quad-2 r<p<\frac{1}{2}, \quad \frac{p+2 r}{1+2 r} \leq q<\frac{p+2 r}{2 p+2 r} .
$$

For our purpose, we need the following.

Lemma 5 ([13]). Let $a, b, d, \theta \in \mathbf{R}$ satisfy $0<a+b, a b=d^{2}$ where $\mathbf{R}$ denotes the set of all real numbers and let

$$
S=\left(\begin{array}{cc}
a & d e^{-i \theta} \\
d e^{i \theta} & b
\end{array}\right) .
$$


Then

$$
S^{p}=(a+b)^{p-1} S \quad \text { for } \quad 0<p .
$$

Now we investigate the optimality of the conditions (I) with $0<p \leq-2 r, \frac{1}{2} \leq$ $p \leq 1$, (II), (III), (IV) by 6 steps.

Step 1. (I) $0<p \leq-2 r$, (II) $0<p<\frac{1}{2}$, (III) $0<p<\frac{1}{2}$.

We show that if $p, q, r$ satisfy

$$
-\frac{1}{2} \leq r<0,0<p<-2 r, 0<q<\frac{p+2 r}{2 r},
$$

then there exist invertible operators $A, B \in B\left(\mathbf{R}^{2}\right)$ with $O \leq B \leq A$ which do not satisfy the inequality (1).

Let

$$
A=\left(\begin{array}{cc}
a & \sqrt{\varepsilon(a-b-\delta)} \\
\sqrt{\varepsilon(a-b-\delta)} & b+\varepsilon+\delta
\end{array}\right)
$$

and let

$$
B=\left(\begin{array}{ll}
1 & 0 \\
0 & b
\end{array}\right)
$$

where

$$
0<b<1<a, 0<\delta=\frac{1-b}{a-1} \varepsilon
$$

as in [13]. Then $O \leq B \leq A$. To show the assertion by contradiction, we assume the inequality (1). We remark

$$
a^{\frac{p+2 r}{q}}, a^{\frac{2 r}{q}} \rightarrow 0 \quad(a \rightarrow \infty) .
$$

Let

$$
U=\frac{1}{\sqrt{\gamma}}\left(\begin{array}{cc}
\sqrt{a-b-\delta} & \sqrt{\varepsilon} \\
\sqrt{\varepsilon} & -\sqrt{a-b-\delta}
\end{array}\right)
$$

where

$$
\gamma=a-b+\varepsilon-\delta
$$

Then $U$ is unitary and

$$
U^{*} A U=\left(\begin{array}{cc}
a+\varepsilon & 0 \\
0 & b+\delta
\end{array}\right)
$$

and hence, by (1) (see [13]), we have

$$
\gamma^{-\frac{1}{q}}\left(\begin{array}{ll}
A_{1} & A_{3} \\
A_{3} & A_{2}
\end{array}\right)^{\frac{1}{q}} \leq\left(\begin{array}{cc}
(a+\varepsilon)^{\frac{p+2 r}{q}} & 0 \\
0 & (b+\delta)^{\frac{p+2 r}{q}}
\end{array}\right)
$$

where

$$
\begin{aligned}
& A_{1}=(a+\varepsilon)^{2 r}\left(a-b-\delta+\varepsilon b^{p}\right), \\
& A_{2}=(b+\delta)^{2 r}\left(\varepsilon+b^{p}(a-b-\delta)\right), \\
& A_{3}=(a+\varepsilon)^{r}(b+\delta)^{r}\left(1-b^{p}\right) \sqrt{\varepsilon(a-b-\delta)} .
\end{aligned}
$$

Let

$$
V=\frac{1}{\sqrt{A_{2}-A_{1}+2 \varepsilon_{1}}}\left(\begin{array}{cc}
\sqrt{\varepsilon_{1}} & \sqrt{A_{2}-A_{1}+\varepsilon_{1}} \\
\sqrt{A_{2}-A_{1}+\varepsilon_{1}} & -\sqrt{\varepsilon_{1}}
\end{array}\right),
$$


where

$$
2 \varepsilon_{1}=-A_{2}+A_{1}+\sqrt{\left(A_{2}-A_{1}\right)^{2}+4 A_{3}^{2}} .
$$

Then $V$ is unitary and

$$
V^{*}\left(\begin{array}{ll}
A_{1} & A_{3} \\
A_{3} & A_{2}
\end{array}\right) V=\left(\begin{array}{cc}
A_{2}+\varepsilon_{1} & 0 \\
0 & A_{1}-\varepsilon_{1}
\end{array}\right) .
$$

Hence

$$
\begin{aligned}
& \varepsilon_{1}\left(\left(A_{2}+\varepsilon_{1}\right)^{\frac{1}{q}}-(a+\varepsilon)^{\frac{p+2 r}{q}} \gamma^{\frac{1}{q}}\right)\left((b+\delta)^{\frac{p+2 r}{q}} \gamma^{\frac{1}{q}}-\left(A_{1}-\varepsilon_{1}\right)^{\frac{1}{q}}\right) \\
& \leq\left(A_{2}-A_{1}+\varepsilon_{1}\right)\left((a+\varepsilon)^{\frac{p+2 r}{q}} \gamma^{\frac{1}{q}}-\left(A_{1}-\varepsilon_{1}\right)^{\frac{1}{q}}\right)\left((b+\delta)^{\frac{p+2 r}{q}} \gamma^{\frac{1}{q}}-\left(A_{2}+\varepsilon_{1}\right)^{\frac{1}{q}}\right)
\end{aligned}
$$

and

$$
\begin{aligned}
& q\left(1-a^{-1}\right)\left(1-b^{p}\right)^{2}\left(1-a^{\frac{p+2 r}{q}} b^{-\frac{p+2 r}{q}}\right)\left(1-a^{\frac{2 r}{q}} b^{-\frac{p+2 r}{q}}\right) \\
& \leq a^{\frac{p+2 r}{q}-2 r} b^{-\frac{p+2 r}{q}+2 p+2 r-1}\left(1-a^{2 r} b^{-p-2 r}\right)\left(1-a^{-\frac{p}{q}}\right) \\
& \quad \times\left\{p(1-b)\left(1-a^{-1} b\right)\left(1-a^{2 r} b^{-p-2 r}\right)\right. \\
& \left.\quad-b^{1-p}\left(1-b^{p}\right)\left(1-a^{-1}\right)\left(1-a^{2 r} b^{-2 r}\right)\right\}
\end{aligned}
$$

by similar arguments as in [13]. Since $\frac{p+2 r}{q}-2 r<0$, we have

$$
0<q\left(1-b^{p}\right)^{2} \leq 0
$$

by letting $a \rightarrow \infty$. This is a contradiction.

Step 2. (I) $\frac{1}{2} \leq p \leq 1$, (II) $-2 r<p \leq 1$.

We show that if $p, q, r$ satisfy

$$
-\frac{1}{2} \leq r<0,-2 r<p \leq 1,0<q<\frac{p+2 r}{1+2 r},
$$

then there exist invertible operators $A, B \in B\left(\mathbf{R}^{2}\right)$ with $O \leq B \leq A$ which do not satisfy the inequality (1). (We remark that, in the condition (I), if $-\frac{1}{4}<r<0$, then $\left\{p \in \mathbf{R} \mid \frac{1}{2} \leq p \leq 1\right\} \subset\{p \in \mathbf{R} \mid-2 r<p \leq 1\}$, and if $r=-\frac{1}{4}, p=\frac{1}{2}$, then the inequality (1) is valid for all $q>0$.) We remark that if the inequality (1) is valid for all invertible operators $A, B \in B(H)$ satisfying $O \leq B \leq A$, then the inequality (1) is valid for all operators $A, B \in B(H)$ satisfying $O \leq B \leq A$.

Let

$$
A=\left(\begin{array}{cc}
2 & 2 \sqrt{c(1-c)} \\
2 \sqrt{c(1-c)} & 4 c
\end{array}\right),
$$

where $0<c<1$ and let

$$
B=\left(\begin{array}{ll}
1 & 0 \\
0 & 0
\end{array}\right) .
$$

Then $O \leq B \leq A$. To show the assertion by contradiction, we assume the inequality (1). Let

$$
V=\left(\begin{array}{cc}
\sqrt{1-c} & \sqrt{c} \\
\sqrt{c} & -\sqrt{1-c}
\end{array}\right) .
$$

Then $V$ is unitary and

$$
V^{*} A V=\left(\begin{array}{cc}
2+2 c & 0 \\
0 & 2 c
\end{array}\right)
$$


By (1), we have

$$
\left(\left(V^{*} A V\right)^{r} V^{*} B^{p} V\left(V^{*} A V\right)^{r}\right)^{\frac{1}{q}} \leq\left(V^{*} A V\right)^{\frac{p+2 r}{q}}
$$

and

$$
\begin{aligned}
& \left(\begin{array}{cc}
(2+2 c)^{2 r}(1-c) & (2+2 c)^{r}(2 c)^{r} \sqrt{c(1-c)} \\
(2+2 c)^{r}(2 c)^{r} \sqrt{c(1-c)} & (2 c)^{2 r} c
\end{array}\right)^{\frac{1}{q}} \\
& \leq\left(\begin{array}{cc}
(2+2 c)^{\frac{p+2 r}{q}} & 0 \\
0 & (2 c)^{\frac{p+2 r}{q}}
\end{array}\right)
\end{aligned}
$$

and, by Lemma 5 , we have

$$
\begin{aligned}
& \delta^{-1}\left(\begin{array}{cc}
(2+2 c)^{2 r}(1-c) & (2+2 c)^{r}(2 c)^{r} \sqrt{c(1-c)} \\
(2+2 c)^{r}(2 c)^{r} \sqrt{c(1-c)} & (2 c)^{2 r} c
\end{array}\right) \\
& \leq\left(\begin{array}{cc}
(2+2 c)^{\frac{p+2 r}{q}} & 0 \\
0 & (2 c)^{\frac{p+2 r}{q}}
\end{array}\right)
\end{aligned}
$$

and hence

$$
0 \leq\left(\begin{array}{cc}
\delta(2+2 c)^{\frac{p+2 r}{q}}-(2+2 c)^{2 r}(1-c) & -(2+2 c)^{r}(2 c)^{r} \sqrt{c(1-c)} \\
-(2+2 c)^{r}(2 c)^{r} \sqrt{c(1-c)} & \delta(2 c)^{\frac{p+2 r}{q}}-(2 c)^{2 r} c
\end{array}\right)
$$

where

$$
\delta^{-1}=\left((2+2 c)^{2 r}(1-c)+(2 c)^{2 r} c\right)^{\frac{1}{q}-1} .
$$

Therefore

$$
\begin{aligned}
0 \leq & \delta^{2}(2+2 c)^{\frac{p+2 r}{q}}(2 c)^{\frac{p+2 r}{q}}-\delta(2 c)^{\frac{p+2 r}{q}}(2+2 c)^{2 r}(1-c)-\delta(2+2 c)^{\frac{p+2 r}{q}}(2 c)^{2 r} c \\
& (2 c)^{\frac{p+2 r}{q}}(2+2 c)^{2 r}(1-c)+(2+2 c)^{\frac{p+2 r}{q}}(2 c)^{2 r} c \leq \delta(2+2 c)^{\frac{p+2 r}{q}}(2 c)^{\frac{p+2 r}{q}}
\end{aligned}
$$

and

$$
\begin{aligned}
& 2^{\frac{p+2 r}{q}}(2+2 c)^{2 r}(1-c) c^{\frac{p+2 r}{q}-1-2 r}+(2+2 c)^{\frac{p+2 r}{q}} 2^{2 r} \\
& \quad \leq\left\{(2+2 c)^{2 r}(1-c)+2^{2 r} c^{1+2 r}\right\}^{1-\frac{1}{q}}(2+2 c)^{\frac{p+2 r}{q}} 2^{\frac{p+2 r}{q}} c^{\frac{p+2 r}{q}-1-2 r} .
\end{aligned}
$$

Since $0<\frac{p+2 r}{q}-1-2 r$, we have

$$
0<2^{\frac{p+2 r}{q}} 2^{2 r} \leq 0
$$

by letting $c \rightarrow+0$. This is a contradiction. (We remark that step 2 is also valid for the condition (I) with $-2 r<p \leq 1$.)

Step 3. (II) $\frac{1}{2} \leq p<-2 r$, (III) $\frac{1}{2} \leq p<1$.

We show that if $p, q, r$ satisfy

$$
-\frac{1}{2} \leq r<-\frac{1}{4}, \frac{1}{2} \leq p<-2 r, 0<q<\frac{p+2 r}{2 p+2 r-1},
$$

then there exist invertible operators $A, B \in B\left(\mathbf{R}^{2}\right)$ with $O \leq B \leq A$ which do not satisfy the inequality (1). 
Define $A, B$ as in step 1 . To show the assertion by contradiction, we assume the inequality (1). Similarly as in step 1, we can show the inequality (7). Since $0<-\frac{p+2 r}{q}+2 p+2 r-1$, we have

$$
0<q\left(1-a^{-1}\right) \leq 0
$$

by letting $b \rightarrow+0$ in (7). This is a contradiction.

Step 4. (I) $1<p$, (II) $1<p$, (III) $1<p$.

We show that if $p, q, r$ satisfy

$$
-\frac{1}{2} \leq r<0,1<p, 0<q,
$$

then there exist invertible operators $A, B \in B\left(\mathbf{R}^{2}\right)$ with $O \leq B \leq A$ which do not satisfy the inequality

$$
B^{\frac{p+2 r}{q}} \leq\left(B^{r} A^{p} B^{r}\right)^{\frac{1}{q}}
$$

or, equivalently,

$$
I \geq B^{\frac{p+2 r}{2 q}}\left(B^{-r} A^{-p} B^{-r}\right)^{\frac{1}{q}} B^{\frac{p+2 r}{2 q}} .
$$

We remark that if the inequality (8) is valid for all invertible operators $A, B \in B(H)$ satisfying $O \leq B \leq A$, then the inequality (8) is valid for all operators $A, B \in B(H)$ satisfying $O \leq B \leq A$. Define $A, B, V$ as in step 2. To show the assertion by contradiction, we assume the inequality (8). Since

$$
I \geq B^{\frac{p+2 r}{2 q}}\left(B^{-r} V V^{*} A^{-p} V V^{*} B^{-r}\right)^{\frac{1}{q}} B^{\frac{p+2 r}{2 q}},
$$

we have

$$
\left(\begin{array}{ll}
1 & 0 \\
0 & 1
\end{array}\right) \geq\left\{(1-c)(2+2 c)^{-p}+c(2 c)^{-p}\right\}^{\frac{1}{q}}\left(\begin{array}{ll}
1 & 0 \\
0 & 0
\end{array}\right) .
$$

Hence

$$
1 \geq(1-c)(2+2 c)^{-p}+c(2 c)^{-p}=(1-c)(2+2 c)^{-p}+2^{-p} c^{1-p} .
$$

Since $1-p<0$, we have $1 \geq \infty$ by letting $c \rightarrow+0$. This is a contradiction.

Step 5. (IV) $1<p$.

We show that if $p, q, r$ satisfy

$$
1<p, 0<q, r<0,
$$

then there exist invertible operators $A, B \in B\left(\mathbf{R}^{2}\right)$ with $O \leq B \leq A$ which do not satisfy the inequality (1) or (2).

First, let $0<p+2 r$. Then the proof is similar to step 2 .

Second, let $p+2 r=0$. Then

$$
\begin{aligned}
\left(A^{r} B^{p} A^{r}\right)^{\frac{1}{q}} \leq A^{\frac{p+2 r}{q}} & \Longleftrightarrow\left(A^{r} B^{-2 r} A^{r}\right)^{\frac{1}{q}} \leq A^{0}=I \\
& \Longleftrightarrow A^{r} B^{-2 r} A^{r} \leq I \\
& \Longleftrightarrow B^{-2 r} \leq A^{-2 r} .
\end{aligned}
$$

The last inequality is valid if and only if $-1 \leq 2 r \leq 0$.

Last, let $p+2 r<0$. We remark that the inequality (2) is equivalent to

$$
B^{\frac{-p-2 r}{q}} \geq\left(B^{-r} A^{-p} B^{-r}\right)^{\frac{1}{q}} .
$$


Define $A, B, V$ as in step 2 . To show the assertion by contradiction, we assume the inequality (9). Since

$$
B^{\frac{-p-2 r}{q}} \geq\left(B^{-r} V V^{*} A^{-p} V V^{*}=B^{-r}\right)^{\frac{1}{q}},
$$

we have

$$
\left(\begin{array}{ll}
1 & 0 \\
0 & 0
\end{array}\right) \geq\left\{(1-c)(2+2 c)^{-p}+c(2 c)^{-p}\right\}^{\frac{1}{q}}\left(\begin{array}{ll}
1 & 0 \\
0 & 0
\end{array}\right) .
$$

The rest of the proof is similar to step 4 .

Step 6. (IV) $0<p \leq 1$.

We show that if $p, q, r$ satisfy

$$
0<p \leq 1,0<q, r<0,
$$

then there exist invertible operators $A, B \in B\left(\mathbf{R}^{2}\right)$ with $O \leq B \leq A$ which do not satisfy the inequality (1).

Define $A, B$ as in step 2 . To show the assertion by contradiction, we assume the inequality (1). Then, similarly as in step 2 , we have

$$
\begin{aligned}
& 2^{\frac{p+2 r}{q}}(2+2 c)^{2 r}(1-c)+(2+2 c)^{\frac{p+2 r}{q}} 2^{2 r} c^{1+2 r-\frac{p+2 r}{q}} \\
& \leq\left\{(2+2 c)^{2 r}(1-c)+2^{2 r} c^{1+2 r}\right\}^{1-\frac{1}{q}}(2+2 c)^{\frac{p+2 r}{q}} 2^{\frac{p+2 r}{q}} \\
& =\left\{(2+2 c)^{2 r}(1-c) c^{-1-2 r}+2^{2 r}\right\}^{1-\frac{1}{q}} c^{(1+2 r)\left(1-\frac{1}{q}\right)}(2+2 c)^{\frac{p+2 r}{q}} 2^{\frac{p+2 r}{q}} .
\end{aligned}
$$

Since

$$
(1+2 r)\left(1-\frac{1}{q}\right)<0<1+2 r-\frac{p+2 r}{q},
$$

we have

$$
0<2^{\frac{p+2 r}{q}} 2^{2 r} \leq 0
$$

by letting $c \rightarrow+0$. This is a contradiction.

\section{ACKNOWLEDGEMENT}

The author would like to express his sincere appreciation to Professor T. Yoshino, the referee and Professor T. Furuta for their valuable comments. Kindly, Professor T. Furuta informed me of the counter-example ([12]) of the Heinz inequality and the referee informed me of the following standard counter-example : $A=\left(\begin{array}{cc}\lambda_{1} & 0 \\ 0 & \lambda_{2}\end{array}\right)$ and $B=\left(\begin{array}{cc}\frac{1}{2} & \frac{1}{2} \\ \frac{1}{2} & \frac{1}{2}\end{array}\right)$ with $\lambda_{i}>0, \lambda_{1} \neq \lambda_{2}$ and $\lambda_{1}^{-1}+\lambda_{2}^{-1}=2$.

\section{REFERENCES}

[1] N. N. Chan and M. K. Kwong, Hermitian matrix inequalities and a conjecture, Amer. Math. Monthly 92 (1985), 533-541. MR 87d:15011

[2] A. Aluthge, Some generalized theorems on p-hyponormal operators, Integr. Equat. Oper. Th. 24 (1994), 497-501. MR 97a:47032

[3] M. Fujii, T. Furuta, E. Kamei, Complements to the Furuta inequality, Journal of the Japan Academy 70 (1994), 239-242. MR 95j:47018

[4] T. Furuta, $A \geq B \geq O$ assures $\left(B^{r} A^{p} B^{r}\right)^{\frac{1}{q}} \geq B^{\frac{p+2 r}{q}}$ for $r \geq 0, p \geq 0, q \geq 1$ with $(1+2 r) q \geq(p+2 r)$, Proc. Amer. Math. Soc. 101 (1987), 85-88. MR 89b:47028

[5] T. Furuta, Two operator functions with monotone property, Proc. Amer. Math. Soc. 111 (1991), 511-516. MR 91f:47023 
[6] T. Furuta, Applications of order preserving operator inequality, Oper. Theory Adv. Appl. 59 (1992), 180-190. MR 94m:47033

[7] T. Furuta, Furuta's inequality and its application to the relative operator entropy, J. Operator Theory 30 (1993), 21-30. MR 95j:47019

[8] T. Furuta, Extension of the Furuta inequality and Ando-Hiai log-majorization, Linear Alg. and its Appl. 219 (1995), 139-155. MR 96k:47031

[9] T. Furuta, Generalized Aluthge transformation on p-hyponormal operators, Proc. Amer. Math. Soc. 124 (1996), 3071-3075. MR 96m:47041

[10] E. Heinz, Beiträge zur Störungstheorie der Spektralzerlegung, Math. Ann. 123 (1951), 415438. MR 13:471f

[11] K. Löwner, Über monotone Matrixfunktionen, Math. Z. 38 (1934), 177-216.

[12] A. W. Marshall and I. Olkin, Inequalities, Theory of Majorization and Its Applications, Academic Press (1979). MR 81b:00002

[13] K. Tanahashi, Best possibility of the Furuta inequality, Proc. Amer. Math. Soc. 124 (1996), 141-146. MR 96d:47025

[14] T. Yoshino, A modified Heinz's inequality, (preprint).

Department of Mathematics, Tohoku College of Pharmacy, Komatsushima, Aoba-ku, SENDAI 981, JAPAN

E-mail address: tanahasi@tohoku-pharm.ac.jp 